\title{
Rotating Regular Black Hole Solution
}

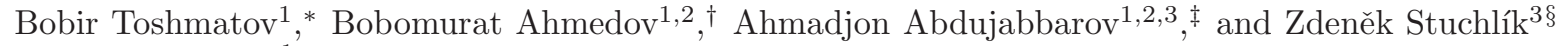 \\ 1 Institute of Nuclear Physics, Ulughbek, Tashkent 100214, Uzbekistan \\ 2 Ulugh Beg Astronomical Institute, Astronomicheskaya 33, Tashkent 100052, Uzbekistan \\ 3 Institute of Physics, Faculty of Philosophy \& Science, Silesian University in Opava, \\ Bezručovo náměstí 13, CZ-74601 Opava, Czech Republic
}

\begin{abstract}
Based on the Newman-Janis algorithm the Ayón-Beato-García spacetime metric [1] of the regular spherically symmetric, static and charged black hole has been converted into rotational form. It is shown that the derived solution for rotating regular black hole is regular and the critical value of the electric charge $Q$ for which two horizons merge into one sufficiently decreases in the presence of nonvanishing angular momentum $a$ of the black hole.

PACS numbers: 04.50.Kd, 04.70.-s, 04.25.-g
\end{abstract}

\section{INTRODUCTION}

It is well known that exact solutions of the Einstein equations have one of the "mysterious" properties of the black hole which is called singularity. Singularity has been considered as one of defects of the general relativity because explanation of singularity can not be made by the general relativity itself. So called regular black hole solutions [1], 2], [3] can be created in order to eliminate singularity from the spacetime metric.

One can distinguish several types of the regular black hole solutions: solutions which are continuous everywhere throughout spacetime; solutions with two simple regions and boundary surfaces joining these regions; solutions with separated two regions: surface layer, thin shell, joining the two regions, etc. The regular black hole solution obtained in the Refs. [1, 2] belongs to the first type of the regular black holes mentioned above.

There exist two types of singularities: the coordinate one (event horizon) and the curvature singularity where the curvature of the manifold is becoming infinite. In the case of coordinate singularity, $g_{r r}$ component of the metric tensor goes to infinity. One can eliminate coordinate singularity by making transformation to the more fortunate coordinate system. Usually by transforming coordinates from the Boyer-Lindquist coordinates to the Eddington-Finkelstein ones one can remove coordinate singularity from the spacetime metric. EddingtonFinkelstein coordinates are based on the freely falling photons. On the other hand, in the curvature singularity, the Riemann tensor components of the spacetime metric diverge. It is impossible to eliminate curvature singularity from the spacetime metric by coordinate transformations.

In the papers [1], 2] and [3], the new regular black hole solutions of the Einstein equations has been found

\footnotetext{
*Electronic address: b.a.toshmatov@gmail.com

${ }^{\dagger}$ Electronic address: ahmedov@astrin.uz

‡Electronic address: ahmadjon@astrin.uz

§Electronic address: zdenek.stuchlik@fpf.slu.cz
}

taking into account the coupling to non-linear electrodynamic field. Afterwards, this solution has been called Ayón-Beato-García regular black hole solution. Recently another regular black hole solution [4] has been considered by introducing a new mass function generalizing the commonly used Bardeen and Hayward mass functions and including the cosmological constant.

The Kerr spacetime metric can be derived from the Schwarzschild one by using the Newman-Janis algorithm [5], [6]. The derivation of the Kerr spacetime metric from Schwarzschild one has been given in several works [5], 7] and [8]. Moreover, in the papers [5] and [8], the KerrNewman solution has been derived from the ReissnerNordström spacetime metric. The Newman-Janis algorithm has been used to derive the radiating KerrNewman black hole in $f(R)$ gravity [9]. The exact nonstatic charged BTZ-like solutions, in $(\mathrm{N}+1)$-dimensional Einstein gravity have been found in [10] in the presence of negative cosmological constant. The Lovelock gravity in the critical spacetime dimension has been studied in Ref. [11].

In order to convert static, spherically symmetric black hole spacetime metric into rotational one (if this spacetime metric is given in the Boyer-Lindquist coordinates $(t, r, \theta, \phi))$ one has to proceed the following five steps of the Newman-Janis algorithm: i) transition from the Boyer-Lindquist coordinates into the advanced Eddington-Filkenstein ones $(u, r, \theta, \phi)$ has to be performed; ii) a null tetrad ( $\mathbf{l}, \mathbf{n}, \mathbf{m}$ and $\overline{\mathbf{m}}$ ) (NewmanPenrose tetrad) for produced metric have to be found; iii) a complex coordinate transformations has to be applied; iv) reverse coordinate transformations into the BoyerLindquist ones have to be done; v) finally, unknown terms of the transformations have to be found based on the reality condition.

Here we convert the static, spherically symmetric Ayón-Beato-García regular black hole spacetime [1], 2], [3] into the rotational one by using the Newman-Janis algorithm [5], [6] and study some of its basic properties. 


\section{NEWMAN-JANIS ALGORITHM TO GET ROTATING REGULAR BLACK HOLE SOLUTION}

In this section we describe the Newman-Janis algorithm which is used for converting the spherically symmetric static black hole spacetime metric into rotational one. The Ayón-Beato-García spacetime metric of the regular spherically symmetric black hole is given as [12]

$$
d s^{2}=-f(r) d t^{2}+\frac{d r^{2}}{f(r)}+r^{2} d \theta^{2}+r^{2} \sin ^{2} \theta d \phi^{2}
$$

where the lapse function $f(r)$ reads

$$
f(r)=1-\frac{2 M r^{2}}{\left(r^{2}+Q^{2}\right)^{3 / 2}}+\frac{Q^{2} r^{2}}{\left(r^{2}+Q^{2}\right)^{2}},
$$

$M$ and $Q$ are the total mass and electric charge of the black hole, respectively. The spacetime metric (1) is the solution of the field equations within general relativity, where the nonlinear electrodynamic field satisfying the weak energy condition is considered as a source. As can be seen from the lapse function (2) the spacetime metric (1) has only the coordinate singularity. This is why in order to remove this singularity one has to write the spacetime metric (11) in the advanced Eddington-Finkelstein coordinates. To do this we make following transformation for incoming photon (or ray):

$$
v=t-r^{*},
$$

and for outgoing photon (or ray)

$$
u=t+r^{*},
$$

where

$$
r^{*}=\int \frac{d r}{f(r)} .
$$

Hereafter, we consider only the outgoing photon (4) case. Then the spacetime metric (1) in the advanced Eddington-Finkelstein coordinates takes the form

$$
d s^{2}=-f(r) d u^{2}-2 d u d r+r^{2} d \theta^{2}+r^{2} \sin ^{2} \theta d \phi^{2} .
$$

The Newman-Penrose tetrad consists of four isotropic vectors $\mathbf{l}, \mathbf{n}, \mathbf{m}$ and $\overline{\mathbf{m}}$. $\mathbf{l}$ and $\mathbf{n}$ are real vectors, $\mathbf{m}$ and $\overline{\mathbf{m}}$ are mutually complex conjugate vectors [8].

Newman-Penrose tetrads satisfy orthogonality condition:

$$
l^{\mu} \cdot m_{\mu}=l^{\mu} \cdot \bar{m}_{\mu}=n^{\mu} \cdot m_{\mu}=n^{\mu} \cdot \bar{m}_{\mu}=0,
$$

and also isotropic condition:

$$
l^{\mu} \cdot l_{\mu}=n^{\mu} \cdot n_{\mu}=m^{\mu} \cdot m_{\mu}=\bar{m}^{\mu} \cdot \bar{m}_{\mu}=0 .
$$

Moreover, the basis vectors usually impose the following normalization condition:

$$
l^{\mu} \cdot n_{\mu}=1, m^{\mu} \cdot \bar{m}_{\mu}=-1,
$$

where $\bar{m}^{\mu}$ is the complex conjugate of $m^{\mu}$.

The contravariant components of the metric tensor of the spacetime metric (6) are

$$
g^{\mu \nu}=\left(\begin{array}{cccc}
0 & -1 & 0 & 0 \\
-1 & f(r) & 0 & 0 \\
0 & 0 & 1 / r^{2} & 0 \\
0 & 0 & 0 & 1 / r^{2} \sin ^{2} \theta
\end{array}\right)
$$

We can rewrite (10) with the help of Newman-Penrose tetrad as

$$
g^{\mu \nu}=-l^{\mu} \cdot n^{\nu}-l^{\nu} \cdot n^{\mu}+m^{\mu} \cdot \bar{m}^{\nu}+m^{\nu} \cdot \bar{m}^{\mu},
$$

where the components of the null tetrad vectors are

$$
\begin{array}{r}
l^{\mu}=[0,1,0,0], n^{\mu}=\left[1,-\frac{1}{2} f(r), 0,0\right], \\
m^{\mu}=\frac{1}{\sqrt{2} r}\left[0,0,1, \frac{i}{\sin \theta}\right], \bar{m}^{\mu}=\frac{1}{\sqrt{2} r}\left[0,0,1,-\frac{i}{\sin \theta}\right] .
\end{array}
$$

As the next step we make the following complex coordinate transformations:

$$
\begin{array}{r}
\tilde{r}=r+i a \cos \theta, \quad \tilde{u}=u-i a \cos \theta, \\
\tilde{\theta}=\theta, \quad \tilde{\phi}=\phi .
\end{array}
$$

As a result of these transformations the components of the null tetrad vectors take the form [7]

$$
\begin{array}{r}
\tilde{l}^{\mu}=[0,1,0,0], \tilde{n}^{\mu}=\left[1,-\frac{1}{2} \tilde{f}(r), 0,0\right], \\
\tilde{m}^{\mu}=\frac{1}{\sqrt{2}(r+i a \cos \theta)}\left[i a \sin \theta,-i a \sin \theta, 1, \frac{i}{\sin \theta}\right], \\
\tilde{\bar{m}}^{\mu}=\frac{1}{\sqrt{2}(r-i a \cos \theta)}\left[-i a \sin \theta, i a \sin \theta, 1,-\frac{i}{\sin \theta}\right],
\end{array}
$$

where the function

$$
\tilde{f}(r)=1-\frac{2 M r \sqrt{\Sigma}}{\left(\Sigma+Q^{2}\right)^{3 / 2}}+\frac{Q^{2} \Sigma}{\left(\Sigma+Q^{2}\right)^{2}}
$$

is the new form of the lapse function (13) and $\Sigma=r^{2}+a^{2} \cos ^{2} \theta$.

Then the metric tensor $g^{\mu \nu}$ takes new $\tilde{g}^{\mu \nu}$ form

$$
\tilde{g}^{\mu \nu}=-\tilde{l}^{\mu} \cdot \tilde{n}^{\nu}-\tilde{l}^{\nu} \cdot \tilde{n}^{\mu}+\tilde{m}^{\mu} \cdot \tilde{\bar{m}}^{\nu}+\tilde{m}^{\nu} \cdot \tilde{\bar{m}}^{\mu}
$$

or

$$
\tilde{g}^{\mu \nu}=\left(\begin{array}{cccc}
\frac{a^{2} \sin ^{2} \theta}{\Sigma \Sigma_{2}} & -1-\frac{a^{2} \sin ^{2} \theta}{\Sigma} & 0 & \frac{a}{\Sigma} \\
-1-\frac{a^{2} \sin ^{2} \theta}{\Sigma} & \tilde{f}(r)+\frac{a^{2} \sin ^{2} \theta}{\Sigma} & 0 & -\frac{a}{\Sigma} \\
0 & 0 & \frac{1}{\Sigma} & 0 \\
\frac{a}{\Sigma} & -\frac{a}{\Sigma} & 0 & \frac{1}{\Sigma \sin ^{2} \theta}
\end{array}\right)
$$

The covariant components of the metric tensor (17) are 


$$
\tilde{g}_{\mu \nu}=\left(\begin{array}{cccc}
-\tilde{f}(r) & -1 & 0 & a(\tilde{f}(r)-1) \sin ^{2} \theta \\
-1 & 0 & 0 & a \sin ^{2} \theta \\
0 & 0 & \Sigma & 0 \\
a(\tilde{f}(r)-1) \sin ^{2} \theta & a \sin ^{2} \theta & 0 & \sin ^{2} \theta\left[\Sigma-a^{2}(\tilde{f}(r)-2) \sin ^{2} \theta\right]
\end{array}\right),
$$

and the spacetime element can be written as

$$
\begin{array}{r}
d \tilde{s}^{2}=g_{u u} d u^{2}+2 g_{u r} d u d r+2 g_{u \phi} d u d \phi+ \\
2 g_{r \phi} d r d \phi+g_{\theta \theta} d \theta^{2}+g_{\phi \phi} d \phi^{2} .
\end{array}
$$

At the following step we turn back into the BoyerLindquist coordinates using, the following transformations:

$$
d u=d t+\lambda(r) d r, d \phi=d \phi+\chi(r) d r
$$

where functions $\lambda(r)$ and $\chi(r)$ are chosen for eliminating $g_{t r}$ and $g_{r \phi}$ terms. Putting (20) into (19) and collecting terms which are corresponding to $g_{t r}$ and $g_{r \phi}$ ones, then equalizing produced expression to zero, one can get two equations for two unknown functions $\lambda(r), \chi(r)$. Solving these equations simultaneously one can find expressions for $\lambda(r)$ and $\chi(r)$ in the following way

$$
\begin{aligned}
& \lambda(r)=-\frac{\Sigma+a^{2} \sin ^{2} \theta}{\Sigma \tilde{f}(r)+a^{2} \sin ^{2} \theta}, \\
& \chi(r)=-\frac{a}{\Sigma \tilde{f}(r)+a^{2} \sin ^{2} \theta} .
\end{aligned}
$$

Finally, the spacetime metric can be expressed in the Boyer-Lindquist coordinates as as

$$
\begin{aligned}
d \tilde{s}^{2}= & -\tilde{f}(r) d t^{2}+\frac{\Sigma}{\Sigma \tilde{f}(r)+a^{2} \sin ^{2} \theta} d r^{2} \\
& -2 a \sin ^{2} \theta(1-\tilde{f}(r)) d \phi d t+\Sigma d \theta^{2} \\
& +\sin ^{2} \theta\left[\Sigma-a^{2}(\tilde{f}(r)-2) \sin ^{2} \theta\right] d \phi^{2},
\end{aligned}
$$

where $\tilde{f}(r)$ is given by equation (15).

If we consider the black hole as non-charged one $(Q=$ 0 ), the lapse function (2) takes the same form of one of the Schwarzschild spacetime metric as well as new spacetime metric (22) converts into the Kerr one, namely

$$
\begin{aligned}
d s^{2}= & -\left(1-\frac{2 M r}{\Sigma}\right) d t^{2}+\frac{\Sigma}{\Delta} d r^{2}-2 \frac{2 M r}{\Sigma} a \sin ^{2} \theta d \phi d t \\
& +\Sigma d \theta^{2}+\left(r^{2}+a^{2}+\frac{2 M a^{2} r \sin ^{2} \theta}{\Sigma}\right) \sin ^{2} \theta d \phi^{2},
\end{aligned}
$$

where $\Delta=r^{2}+a^{2}-2 M r, \sum=r^{2}+a^{2} \cos ^{2} \theta$.

In order to investigate properties of the spacetime metric (22) we here study the behavior of the $g_{r r}$ and $g_{t t}$ components of the spacetime metric (22).

\section{PROPERTIES OF ROTATING REGULAR BLACK HOLE SOLUTION}

Now we will analyze the static limit and event horizon defined by the conditions $g_{t t}=0$ and $1 / g_{r r}=0$, respectively.

Obtained new spacetime metric (22) is also regular everywhere. From the Fig. 1 and Fig. 2 one can easily see that for some set of values of the rotation parameter $a$ and electric charge $Q$ the solution (22) has coordinate singularity (event horizon).

The radial dependence of the function $\tilde{f}(r)$ presented in Fig. 1 and Fig. 2 show that with increase of the value of the rotation parameter $a$ and charge $Q$ possibility of existence of the horizon decreases. In the equatorial plane $(\theta=\pi / 2)$ the dependence of the function $\tilde{f}(r)$ on the rotation parameter $a$ vanishes and the existence of the horizon depends only on the value of the charge $Q$.

There is critical value of the charge $Q$ for which two surface described by the solutions of the condition $g_{t t}=0$ merge into one. In order to find the critical value of $Q$ the lapse function $g_{t t}(r, a, \theta, Q)$ must satisfy the couple of conditions:

$$
g_{t t}(r, a, \theta, Q)=0, \partial_{r} g_{t t}(r, a, \theta, Q)=0 .
$$

Since the lapse function $g_{t t}$ is the function of four quantities $r, a, \theta$ and $Q$, solving the equations (24) with respect to $r$ and $Q$ one can get the solution as a function of $a$ and $\theta$. In Fig. 3 dependence of the critical value of the electric charge $Q$ and radius of the static limit surface $r$ which is corresponding to critical state on the rotation parameter $a$ have been shown for several values of $\theta$. The shaded region in the $Q-a$ plot corresponds to the regular black hole with the static limit. The unshaded region in the $Q-a$ plot corresponds to the regular black hole without static limit. The $r-a$ plot represents the dependence of the radius of the static limit on the rotation parameter which corresponds to the border of shaded-unshaded regions. In order to find the same critical value of the charge one may use the conditions

$$
g_{r r}(r, a, \theta, Q)=0, \partial_{r} g_{r r}(r, a, \theta, Q)=0 .
$$

In Fig. 4 dependence of the critical value of the electric charge $Q$ and radius of the static limit surface $r$ which is corresponding to the critical state on the rotation parameter $a$ have been shown for several values of $\theta$. The shaded region in the $Q-a$ plot corresponds to the regular black hole with two (outer and inner) event horizons. 

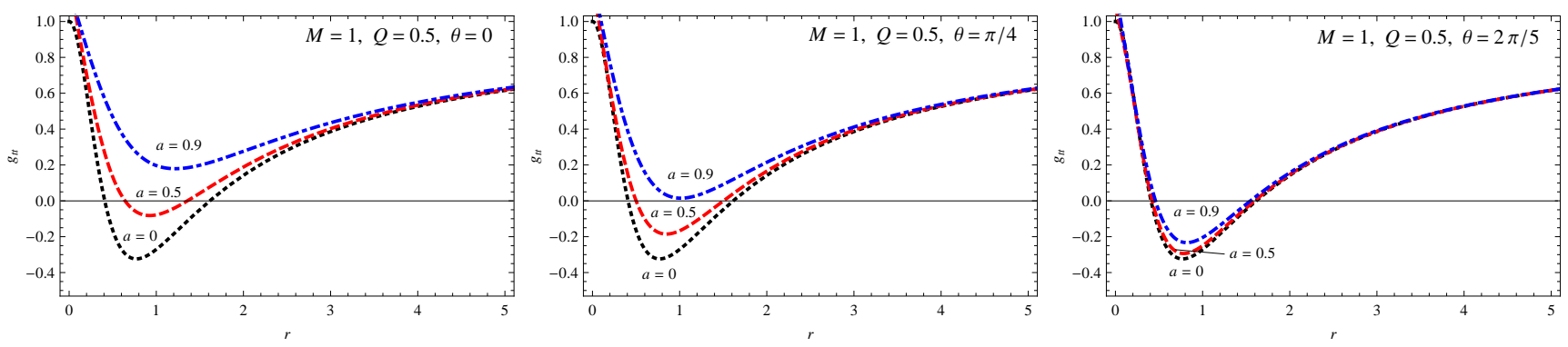

FIG. 1: The dependence of the $g_{t t}$ component of the metric tensor from the radial coordinate $r$ for the typical values of the rotation parameter $a$.
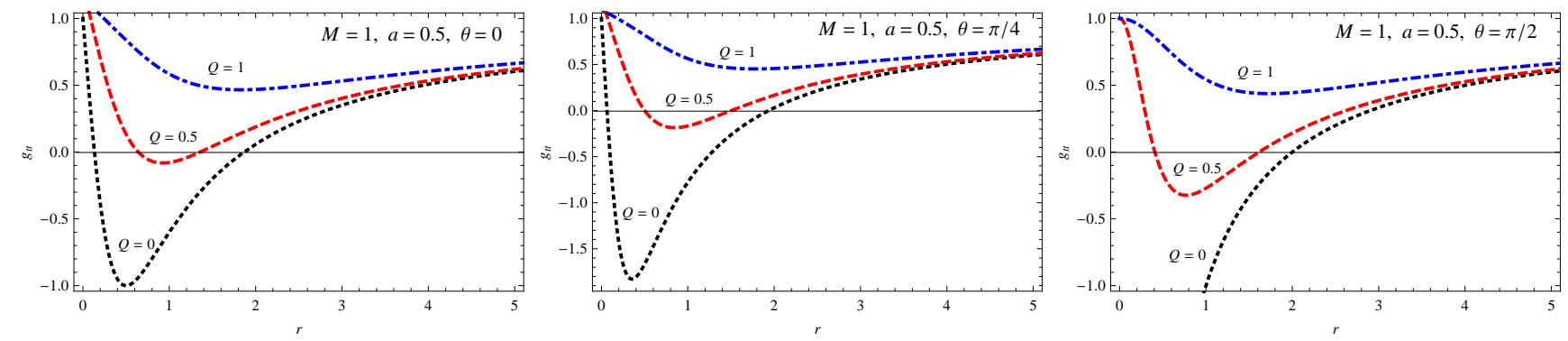

FIG. 2: The dependence of the $g_{t t}$ component of the metric tensor from the radial coordinate $r$ for the typical values of the electric charge $Q$.
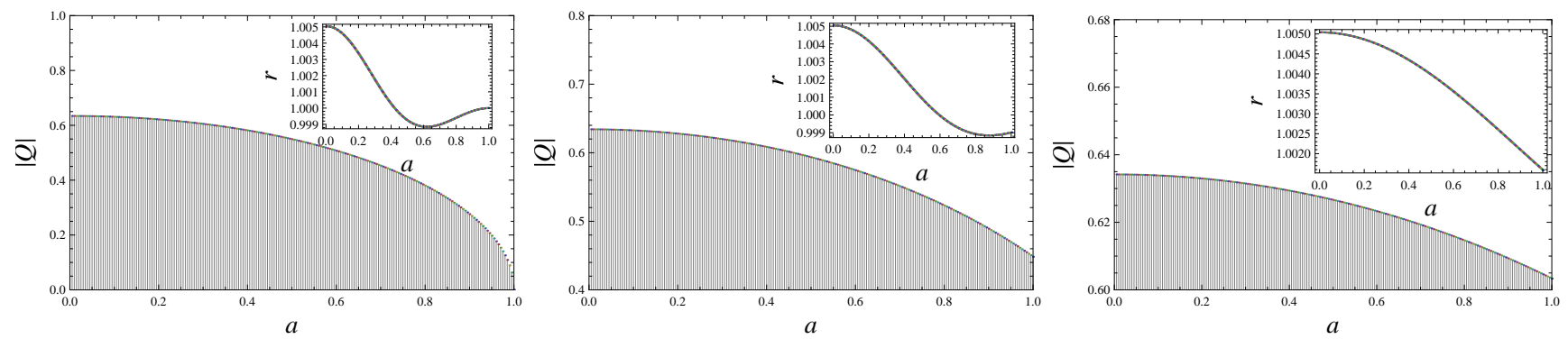

FIG. 3: The dependence of the critical value of the electric charge $Q$ and radius of the horizon $r$ from the rotation parameter $a$ for the different values of $\theta: \theta=0, \theta=\pi / 4$ and $\theta=2 \pi / 5$ (from the left to right, respectively).
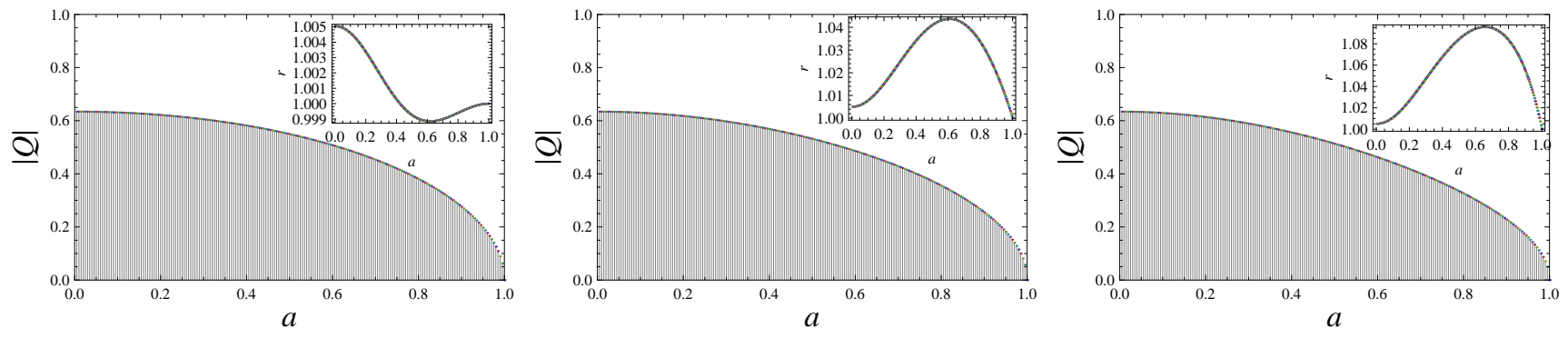

FIG. 4: The dependence of the critical value of the electric charge $Q$ and radius of the event horizon $r$ from the rotation parameter $a$ for the different values of $\theta: \theta=0, \theta=\pi / 4$ and $\theta=\pi / 2$ (from the left to right, respectively).

The unshaded region in the $Q-a$ plot corresponds to the

regular black hole without event horizon. The $r-a$ plot 
represents the dependence of the radius of the static limit on the rotation parameter which corresponds to the border of shaded-unshaded regions. The regular black hole with the rotation parameter $a \sim 1$ can have the horizon in the poles of the black hole $(\theta=0, \pi)$ even in the case when the value of the charge $Q$ is very small. One may conclude that in the presence of the rotation parameter the small value of electric charge may cause the elimination of the singularity.

The critical values of the electric charge $Q_{\mathrm{cr}}$ are different for the static limit and the event horizon. The critical value of the electric charge for event horizon is more rapidly decreasing with the increase of the rotation parameter $a$ with compare to that for the static limit. This means that the event horizon disappears earlier with the increase of the electric charge for the fixed value of the rotation parameter $a$.

One can see from Fig. 3 and 4 that when the value of the electric charge $Q \leq 0.633$ the static black hole $(a=0)$ has horizon. If $Q \leq 0.605$ even extreme black hole can have the horizon in the equatorial plane $(\theta=\pi / 2)$.

Fig. 5 provides the shape and size of the ergoregion in the $x-z$ plane where $z=r \cos \theta$ and $z=r \sin \theta$. With the increase of the electric charge one can observe the increase of the relative shape and size of the ergosphere. Note that for the values of electric charge with $Q \geq Q_{\text {cr }}$ the event horizon and static limit both disappear.

As the further step we study the question of satisfying the weak energy condition and choose the Locally non rotating frame (LNRF) in order to get the stress-energy tensor in diagonal form, namely, $T^{\alpha \beta}=\left(\rho, P_{1}, P_{2}, P_{3}\right)$. Then the weak energy condition reads as [6] $\rho \geq 0, \quad \rho+$ $P_{i} \geq 0$, where $i=1,2,3$.

Finally, we express the spacetime geometry in frame of the LNRF and study the behavior of the angular velocity of these frames. The orthonormal tetrad of the LNRF has the following form

$$
\begin{aligned}
\omega^{t} & =\left|g_{t t}-g_{\phi \phi} \Omega_{\mathrm{LNRF}}^{2}\right|^{1 / 2} d t, \\
\omega^{r} & =\left|g_{r r}\right|^{1 / 2} d r, \\
\omega^{\theta} & =\left|g_{\theta \theta}\right|^{1 / 2} d \theta, \\
\omega^{\phi} & =\left|g_{\phi \phi}\right|^{1 / 2} d \phi-\left|g_{\phi \phi}\right|^{1 / 2} \Omega_{\mathrm{LNRF}} d t,
\end{aligned}
$$

where

$$
\Omega_{\mathrm{LNRF}}=\frac{a(1-\tilde{f}(r, \theta))}{\Sigma-a^{2}(\tilde{f}(r, \theta)-2) \sin ^{2} \theta}
$$

is the angular velocity of the LNRF frame.

In Fig. 6 the 3D plot of the radial and angular dependence of density and pressures is shown for the given value of the rotation parameter $a / M=0.5$ and electric charge $Q / M=0.9$. One can easily see that the weak energy condition is violated near the nonsingular origin of rotating regular black hole.

\section{CONCLUSION}

In this paper, we used a regular black hole solution with the source with the nature of nonlinear electrodynamics obtained by Ayón-Beato and García [1] 3] to generate rotating regular black hole solution which includes the Ayón-Beato-García and Kerr metrics as special cases. Considered Newman-Janis algorithm uses static solution to generate rotating solutions without touching the field equation and very useful in order to get rotating black hole solutions.

The relation between Einstein vacuum solution and any non vacuum solution of general relativity opens new direction in studying the properties of the new solution with nonlinear electrodynamic source. Obviously, when electric charge is vanishing, the solution reduces to the vacuum one. Here we have obtained an exact rotating regular $\mathrm{BH}$ solution in the framework of general relativity. Obtained solution gives opportunity to study the geometrical and causal structures, as well as test particles motion around rotating regular $\mathrm{BH}$, which will be subject of the future projects. On the other hand, it could be also very interesting to compare the rotating Ayon-Beato-Garcia regular spacetimes without horizons to the Kerr naked singularity spacetimes, testing if the interesting and unusual physical phenomena occuring in the Kerr naked singularity spacetimes [14] could arise in the regular rotating spacetimes too.

\section{Acknowledgments}

The authors acknowledge the project Supporting Integration with the International Theoretical and Observational Research Network in Relativistic Astrophysics of Compact Objects, CZ.1.07/2.3.00/20.0071, supported by Operational Programme Education for Competitiveness funded by Structural Funds of the European Union. One of the authors (ZS) acknowledges the Albert Einstein Center for gravitation and astrophysics supported by the Czech Science Foundation No. 14-37086G. A.A. and B.A. thank the Goethe University, Frankfurt am Main, Germany and Faculty of Philosophy and Science, Silesian University in Opava (Czech Republic) for the warm hospitality. This research is supported in part by Projects No. F2-FA-F113, No. EF2-FA-0-12477, and No. F2-FA-F029 of the UzAS and by the ICTP through the OEA-PRJ-29 and the OEA-NET-76 projects and by the Volkswagen Stiftung (Grant No. 86 866). 

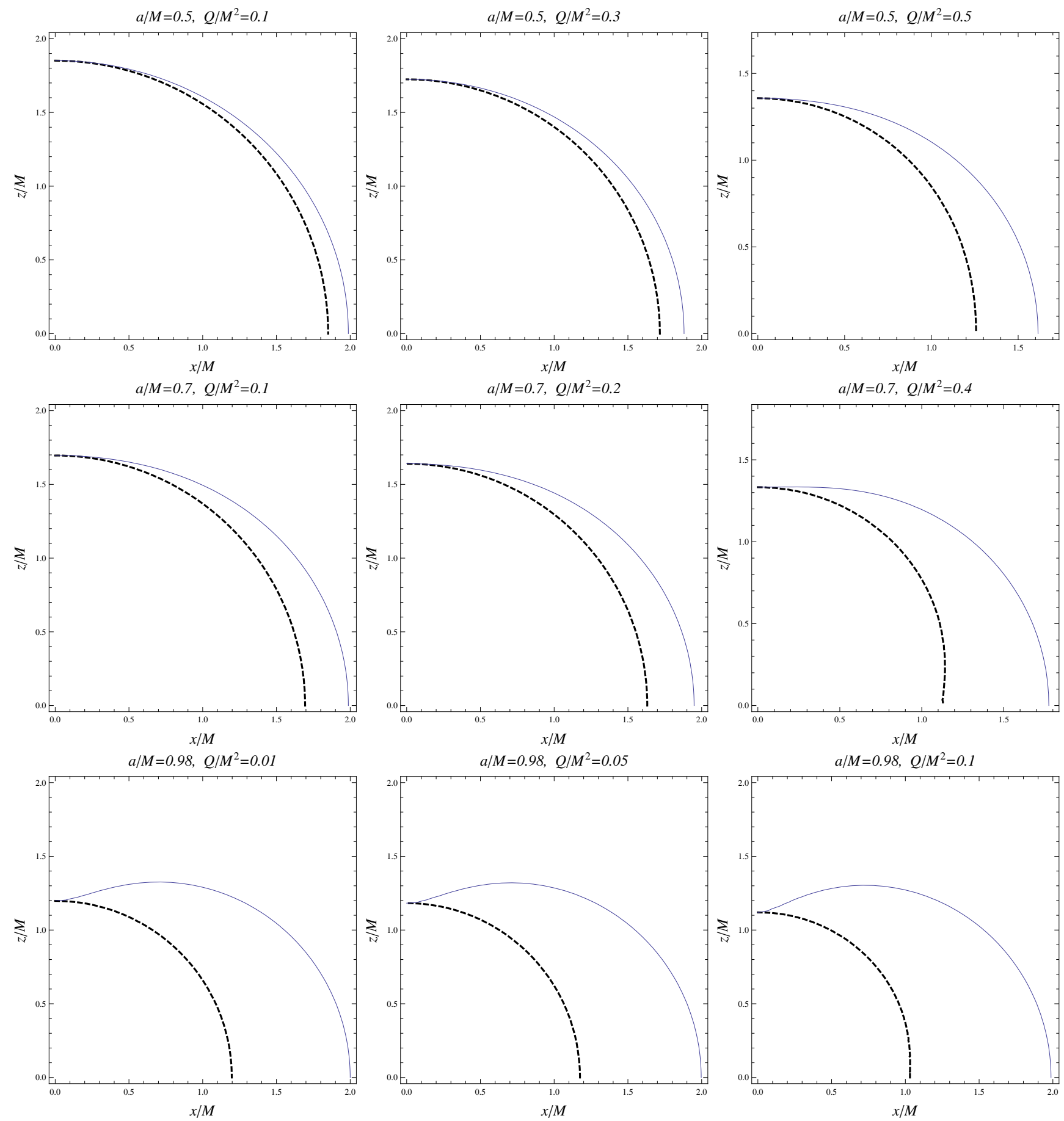

FIG. 5: The shape and size of the ergosphere for the different values of the rotation parameter $a$ and electric charge $Q$.

[2] E. Ayón-Beato, A. García, Phys. Rev. Lett. B 464, 25 (1999).

[3] E. Ayón-Beato, A. García, Gen. Rel. Grav. B 31, 629 (1999).

[4] J.C.S. Neves, A. Saa, arXiv:1402.2694 (2014).

[5] S.P. Drake, P. Szekeres, Gen. Rel. Grav., 32, 445 (2000).

[6] C. Bambi, L. Modesto, Phys. Lett B 721, 329 (2013).

[7] S.P. Drake, R. Turolla, Class. Quan. Grav., 14, 1883
(1997).

[8] J.C. Lombardo, Diego, Class. Quan. Grav., 21, 1407 (2004).

[9] S.G. Ghosh, S.D. Maharaj, U. Papnoi, Eur. Phys. J. C 73, 2473 (2013).

[10] S. G. Ghosh, Int. J. Mod. Phys. D, 21, 1250022 (2012).

[11] N. Dadhich, S.G. Ghosh, S. Jhingan, Phys. Lett. B, 711, 196 (2012). 

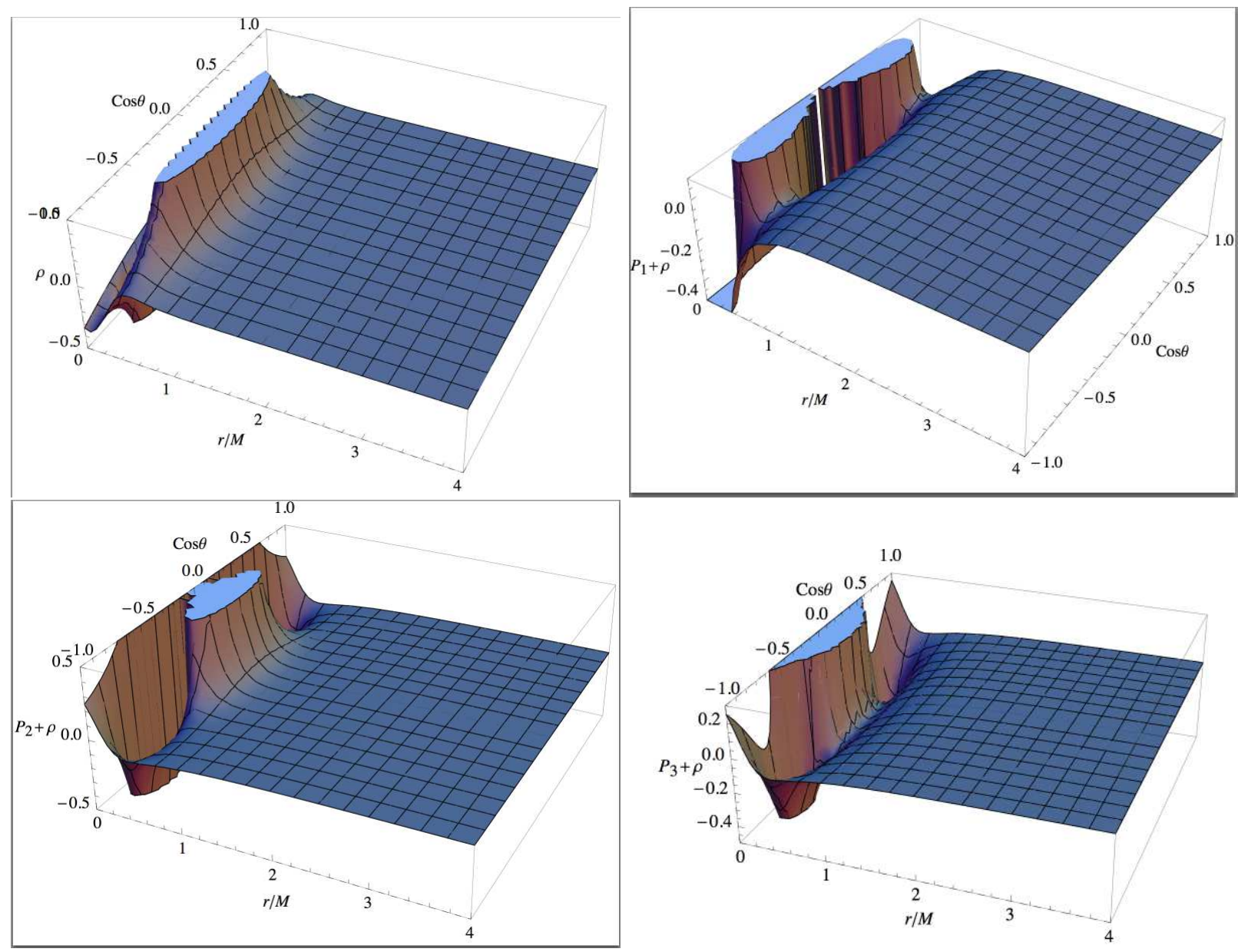

FIG. 6: The radial and angular dependence of $\rho, P_{1}+\rho, P_{2}+\rho$, and $P_{3}+\rho$ for the given value of the rotation parameter $a / M=0.5$ and electric charge $Q / M=0.9$.

[12] A. Garcia, E. Hackmann, J. Kunz, C. Lämmerzahl, A. Macias, arXiv:1306.2549 (2013).

[13] R. Ferraro, arXiv:1311.3946 (2013).

[14] Z. Stuchlík, J. Schee, Class. Quantum Grav., 27, 215017
(2010); M. Patil, P.S. Joshi, Phys. Rev. D, 84, 104001 (2011); Z. Stuchlík, J. Schee, Class. Quantum Grav., 30, $075012(2013)$. 